\title{
Positional uncertainty in optical-based metrology systems in large volume manufacturing
}

\author{
Ainsley Miller ${ }^{1}$, Anthony J. Mulholland ${ }^{2}$, Stephen Gareth Pierce ${ }^{1}$, Ben Hughes ${ }^{3}$ and Alistair B. Forbes ${ }^{3}$ \\ ${ }^{1}$ Department of Electronic and Electrical Engineering, University of Strathclyde, Glasgow, United Kingdom, G1 1RD \\ ${ }^{2}$ Department of Mathematics and Statistics, University of Strathclyde, Glasgow, United Kingdom, G1 1 XH \\ ${ }^{3}$ National Physical Laboratory (NPL), Teddington, London, United Kindgom, TW11 OLW
}

\begin{abstract}
Modern manufacturing increasingly utilizes automated systems for component positioning and assembly and a vital aspect of autonomous precision manufacturing is large volume metrology. One popular approach uses light rays, which travel through the volume of air, to calculate the position of an object of interest. These optical-based metrology systems such as photogrammetry and laser tracking are crucial in improving the accuracy and quality associated with robotic assembly. However, in an industrial setting, these positional measurement systems give rise to uncertainties which can in many instances be greater than the required tolerances. One source of this uncertainty is light refraction due (in part) to temperature fluctuations in the air. This paper will describe how COMSOL Multiphysics can be used to create simulated data with complete ground truth on the refractive index field in the volume, and will discuss the measurement uncertainties associated with these simulations. Over industry relevant distances (tens of metres) the uncertainty in the position measurement due to light refraction is of the order of a millimetre.
\end{abstract}

\section{Section: RESEARCH PAPER}

Keywords: photogrammetry; laser tracker; large volume; refraction; uncertainty

Citation: Ainsley Miller, Anthony J. Mulholland, Stephen Gareth Pierce, Ben Hughes and Alistair B. Forbes, Positional uncertainty in optical-based metrology systems in large volume manufacturing, Acta IMEKO, vol. 7, no. 2, article 14, June 2018, identifier: IMEKO-ACTA-07 (2018)-02-14

Section Editor: Franco Pavese, Italy

Received December 12, 2017; In final form April 6, 2018; Published June 2018

Copyright: ( $\odot 2018$ IMEKO. This is an open-access article distributed under the terms of the Creative Commons Attribution 3.0 License, which permits unrestricted use, distribution, and reproduction in any medium, provided the original author and source are credited

Funding: This work is funded by a Strathclyde University and National Physical Laboratory (NPL) Studentship

Corresponding author: Ainsley Miller, email: ainsley.miller@strath.ac.uk

\section{INTRODUCTION}

As the world enters the fourth industrial revolution, the autonomous age, automated systems are being increasingly used for component positioning and assembly [1]. Industries are interested in autonomous and robotic manufacturing as it potentially reduces costs and increases productivity [2]. The robots used in large volume manufacturing need to know their own spatial position in the working volume and the spatial position of the object that they are working on. One possible method for achieving this "local GPS" system is using lightbased sensors.

Large volume metrology involves using light rays which travel through the air volume to gather data on the distance or angle between the measurement apparatus and the object being measured [3]. Popular optical-based metrology systems such as photogrammetry and laser trackers are crucial in improving the accuracy and quality associated with robotic assembly [4]. Photogrammetry systems can simply be thought of as systems which use photographs to calculate angles between the camera and the reflector [5] and a laser tracker uses laser beams to measure both the angle and the distance from the tracker to the reflector [4], [6].

In an industrial setting these positional uncertainties can in many instances be greater than the required tolerances. One source of uncertainty that arises when considering large scale industrial settings is light refraction (bending of the light ray path) due to, for example, temperature fluctuations in the air; these fluctuations create a heterogeneous refractive index map. As photogrammetry systems and laser trackers both use angular based measurements to determine coordinates, they are both affected by refraction. This source of uncertainty could contribute to difficulties in deploying robotic systems in high 
precision industrial settings. There are of course other sources of uncertainty in optical based metrology systems, but refractive effects in the air volume will form the focus of the work in the paper. Therefore, accounting for the refractive index effects of air is important to improving positional accuracy and this is a long standing and open problem in large volume metrology.

The impact that the fluctuations of the refractive index in air has on industrial measurements can be significant. The recently completed international collaboration on the project "Large volume unified metrology for industry and novel applications and research (LUMINAR)" [6] focused on the refractive index effects in an industrial setting. They found that in a typical aircraft industrial space the positional measurement uncertainty can be significant [7]. In addition, [8] found that a light beam will deviate from a straight line by $0.2 \mathrm{~mm}$ for every $15 \mathrm{~m}$ it travels horizontally when there is a vertical temperature gradient of approximately $2 \mathrm{~K} / \mathrm{m}$ (and a vertical height of $6 \mathrm{~m}$ ). Additionally, it was found that in a working volume of $100 \mathrm{~m}^{3}$ the average positional error of the VICON T160 photogrammetry system was millimetre scale [9].

This paper will look first at characterising the errors associated with optical-based metrology systems due to refraction. The following section will present the methodology used by simulation software to examine the effect that varying parameters that characterise a temperature profile in the air volume have on the positional error. Following that the results of the simulations will be presented and discussed. The paper will conclude by summarising the work done and future directions.

\section{CHARACTERISING THE ERRORS IN OPTICAL- METROLOGY SYSTEMS}

Let us start by defining the positional error as the Euclidean distance between the true (known in the calibration stage) position and the estimated position of the reflector. Opticalbased metrology systems assume that the volume of air is homogeneous and that light rays travel in straight lines (so no refractive effects).

In addition to an angular measurement, the laser tracker calculates the distance (that is, the distance as perceived by the laser tracker) of the light ray from the laser tracker $\left(l_{i}\right)$ to the reflector $\left(r_{j}\right)$ (denote this distance by $d_{i j}$ ). This distance can be used to produce a straight line (see the straight dashed lines in Figure 1). If we restrict attention to just distance measurements then, in a two dimensional plane, one can then deploy two laser trackers and use the intersection point of the two straight rays to estimate the position of the reflector $r_{i}^{E}$. The error in the estimated position is shown in Figure 1 and is denoted by $\varepsilon_{1}^{l} \quad$ where $\varepsilon_{1}^{l}=\left|r_{j}-r_{i}^{E}\right|$. These errors will typically be much smaller than the errors due to angular based calculations.

Similarly, the photogrammetry system calculates the angle (that is, the angle as perceived by the camera denoted by $\theta_{i j}$ ) that the line from the camera $\left(c_{i}\right)$ to the reflector $\left(r_{j}\right)$ makes with the normal to the camera plane. This angle can also be used to produce a straight line (see the straight dashed lines in Figure 2). In a two-dimensional plane one can then deploy two cameras and use the intersection point of the two straight rays to estimate the position of the reflector $r_{i}^{E}$. The error in the estimated position is as shown in Figure 2 and is denoted by $\varepsilon_{1}^{c}$

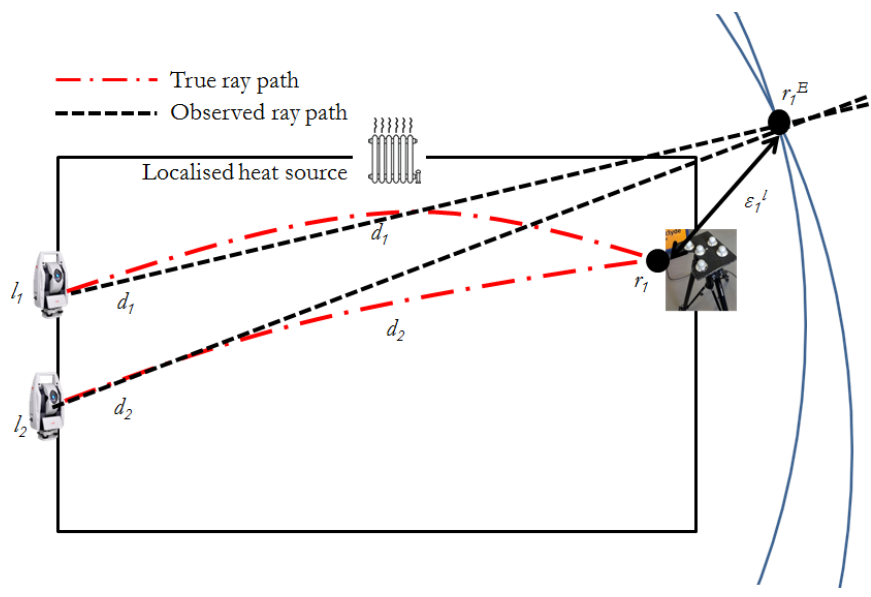

Figure 1. Figure showing the errors caused by refraction of the light rays when using just the distance measurement in the laser tracker. Here we have two laser trackers $\left(I_{1}\right.$ and $\left.l_{2}\right)$ tracking a reflector $r_{1}$. The red dashed lines show the true ray path where the light rays bend towards the localised heat source and have distance $d_{1}$ and $d_{2}$ respectively. The black lines show the equivalent straight ray path that the laser trackers use in their calculations since this assumes the volume is homogeneous (has a constant refractive index throughout and hence no refraction takes place). The intersection of the curved solid lines therefore gives the estimated position of the reflector $\left(r_{1}{ }^{E}\right)$. The positional error is then given by $\varepsilon_{1}{ }^{\prime}=\left|r_{1}-r_{1}{ }^{E}\right|$.

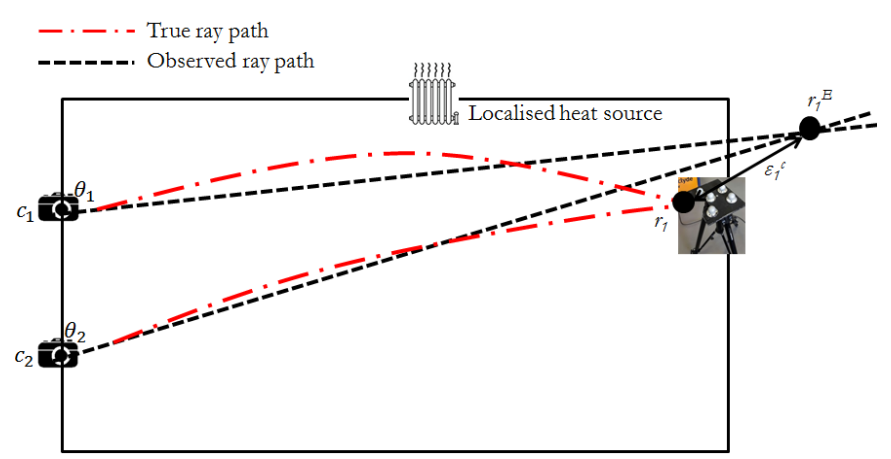

Figure 2. Figure showing the errors caused by refraction of the light rays when using the photogrammetry (angular) system. Here we have two cameras $\left(c_{1}\right.$ and $\left.c_{2}\right)$ tracking reflector $r_{1}$. The red dashed lines show the true ray path where the light rays bend towards the localised heat source and have initial angles $\theta_{1}$ and $\theta_{2}$ (this is the angle that the line from camera to the reflector makes with the normal to the camera plane). The black lines show the equivalent straight ray path that the photogrammetry systems use in their calculations since this assumes the volume is homogeneous (has a constant refractive index throughout and hence no refraction takes place). The intersection of the dashed black lines therefore gives the estimated position of the reflector $\left(r_{1}{ }^{E}\right)$. The positional error is then given by $\varepsilon_{1}^{\prime}=\mid r_{1}$ $r_{1}{ }^{E}$.

where $\varepsilon_{1}^{c}=\left|r_{j}-r_{i}^{E}\right|$. The remainder of this paper will focus mainly on the photogrammetry case, but all the work is transferrable to the laser tracker case.

\section{COMSOL MODELLING}

As a precursor to experiments and to allow us to explore the relationship between the measurement uncertainties and the underlying temperature field a virtual simulation of a large volume light-based metrology setting was constructed wherein spatial variations in the refractive index of the air volume can 
be prescribed. This carefully controlled environment will supply the ground truth needed to accelerate the development of algorithms and methodologies ahead of their experimental deployment. The forward model (ray-tracing) was carried out using simulations in COMSOL Multiphysics ${ }^{\circledR}$ 5.3 Ray Tracing toolbox [10] and for computational expediency the investigation was restricted to a two dimensional plane (it can very simply be extended to three dimensions in COMSOL).

It is clear from these simulations that gradients in the refractive index that are orthogonal to the light ray's path dominate the resulting measurement uncertainties. In other words, it is not sufficient to simply consider a light ray traversing a series of layers that have constant but differing refractive indices; the refraction in this case would simply follow Snell's law. It is important to include the transverse gradients and this is well documented [11].

To mimic the presence of a single source of heat a Gaussian temperature profile was placed in the plane containing the light ray (so we are assuming that as the light ray refracts and bends that it remains within this plane) via

$$
T(x, y)=\left(T_{\max }-T_{\min }\right) \exp \left(\frac{\left(x^{2}+y^{2}\right)}{\sigma^{2}}\right)+T_{\min }
$$

where $x$ and $y$ give the co-ordinates of the point in the plane, $\sigma$ is the variance of the distribution measured in metres, $T_{\max }$ is the maximum temperature that the domain can take and similarly $T_{\min }$ is the minimum temperature that the domain takes ( $T_{\min }$ was kept at $295 \mathrm{~K}$ throughout and both $T_{\max }$ and $T_{\min }$ are measured in Kelvins).

The refractive index is a function of many atmospheric parameters such as temperature, humidity, pressure and carbon dioxide concentration as captured via the Ciddor equation [12][14]. COMSOL requires the refractive index map in order to define the material properties, so the temperature values in (1) were transformed to a refractive index map using the Ciddor equation [14]; all other parameters in the equation were set at the NIST average values [15].

The COMSOL simulation will shortly be used to examine the relationship between the resulting positional uncertainty and the initial ray angle, the value of $T_{\max }$ and the spread of the temperature distribution (by varying $\sigma$ in (1)), but first the simulation framework will be described.

\subsection{Setting up the Simulation in COMSOL}

All the simulations described in this paper were carried out in two dimensions and the COMSOL Model Wizard was used to create each new simulation. In all of the simulations the size of the domain was kept constant. The size chosen was in keeping with a laboratory scale photogrammetry area; $3 \mathrm{~m}$ in the horizontal $(x)$ direction and $4 \mathrm{~m}$ in the vertical $(y)$ direction. A rectangular domain was created in COMSOL - it should be noted that the bottom left corner of the domain has coordinates $(-1.5,-2) \mathrm{m}$ such that the hottest part of the domain can be positioned at the origin (see Figure 3). The next step was to input the temperature profile as defined in (1). This was achieved by entering the component option in the Model Builder window and adding a new variable in the definitions section. Once in this menu, the right-hand side of (1) was input into the Expression box and this variable was named as T_domain. The

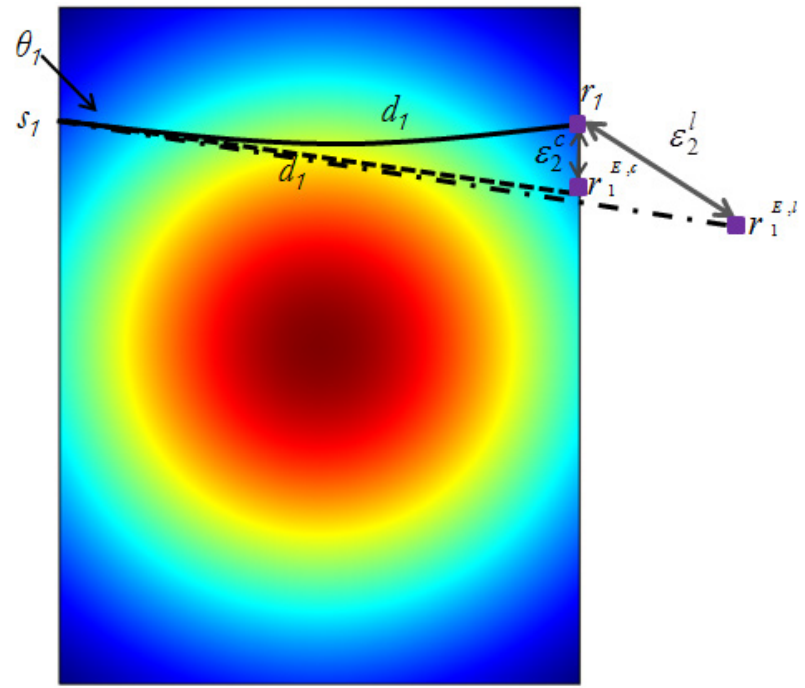

Figure 3. Figure showing the errors caused by refraction of the light rays caused by a Gaussian temperature profile. The size of the volume is 3 metres in the horizontal direction and 4 meters in the vertical direction. Here we have an optical-based metrology sensor positioned at $S_{1}$ (this can be either the photogrammetry system or the laser tracker) which is tracking a reflector positioned at $r_{1}$. The temperature profile in this diagram causes the light ray to bend towards the heat source (the red colours) as shown by the solid black line; where the ray has initial angle $\theta_{1}$ (this is the angle that the line from camera to the reflector makes with the normal to the camera plane) and total distance $d_{1}$. When $S_{1}$ is the photogrammetry system the dashed black line shows the equivalent straight ray path that the photogrammetry system uses in its calculations since this assumes the volume is homogeneous (has a constant refractive index throughout and hence no refraction takes place). The intersection of the dashed black line and the back wall therefore gives the estimated position of the reflector $\left(r_{1}{ }^{E}\right.$ $\left.{ }^{c}\right)$. The positional error is then given by $\varepsilon_{2}^{c}=\left|r_{1}-r_{1}^{E, c}\right|$. Similarly, when $S_{1}$ is the laser tracker, the dot dashed lines show the equivalent straight ray path that the laser tracker uses in its calculations since this assumes the volume is homogeneous, as in the photogrammetry system. The laser tracker gives an angular component and a distance component as output that gives an estimated position of the reflector $\left(r_{1}^{E, I}\right)$. The positional error is then given by $\varepsilon_{2}^{\prime}=\left|r_{1}-r_{1}^{E, I}\right|$.

next step was to convert the temperature map into a refractive index map. This was achieved by selecting an interpolation from the function menu of the global definitions functionality. Once in this menu we named our function $n \_$int and input temperatures ranging from $293.15 \mathrm{~K}$ to $323.15 \mathrm{~K}$ in the column labelled $t$. In order to fill in the $f(t)$ column the refractive index values were calculated for each of the temperatures using the Ciddor equation [14], [15]. To ensure that these refractive index values were used in the model the Geometrical Optics (gop) functionality was accessed. In this functionality the Medium Properties option was located and in the Medium Properties heading the Refractive index real part was defined as user defined from the drop-down menu. Finally, the refractive index was defined to take the values of n_int(T_domain).

Following this, the rays propagating within this geometry had to be defined. In the Geometrical Optics (gop) functionality of the Model Builder the Release from Grid option was used. This allows the user to prescribe the start point of a ray and its directional vector, and one is needed for each ray which propagates within the domain.

In the first study 25 rays were simulated with each one having a different initial direction but each one starting from the same point $(-1.5,0) \mathrm{m}$. The vector describing the light rays' initial 
direction is $x_{0}=\left[1, y_{0}\right]$ where $y_{0}$ takes the values $0, \pm 0.05, \pm$ $0.10, \pm 0.15, \ldots, \pm 0.6$. The upper limit here is 0.6 as any higher values lead to the light ray not reaching the other side of the domain, i.e. having an end co-ordinate as $(1.5, y) \mathrm{m}$ with $y \in[-2,2]$. Figure 4 shows the 25 rays propagating in the geometry. In this case, the values of $T_{\max }$ and $\sigma$ were kept constant at $314 \mathrm{~K}$ and $1.3107 \mathrm{~m}$ respectively. The value for $\sigma$ was chosen at it ensures that at the four corners of the domain the temperature is exactly $T_{\min }$.

The second simulation examined the impact that $T_{\max }$ has on the positional error. In this case the number of rays propagating through the geometry was reduced to one. The chosen ray was the one whose initial ray angle led to the biggest positional error in the first set of simulations. All other parameters in this case remain as before apart from $T_{\max }$. In these simulations $T_{\max }$ takes values from $299 \mathrm{~K}$ to $374 \mathrm{~K}$.

The final simulations examined the effect that varying the spread of the temperature profile $(\sigma)$ has on the positional uncertainty. As in the second set of simulated experiments there was again only one ray propagating through the geometry (in fact, the same ray which was used in the previous case). In this case $T_{\max }$ is set as $314 \mathrm{~K}$ as in the first case and the values for $\sigma$ range from 0.5 to $3.5 \mathrm{~m}$. In all of the simulations the output was a set of $(x, y)$ co-ordinates for each ray.

\section{RESULTS OF COMSOL SIMULATIONS}

In order to analyse the results, we consider Figure 3 which shows how the positional uncertainties (errors) were defined. This paper only considers the results from the error in the photogrammetry system, $\varepsilon_{2}^{c}$. Firstly assume that there is a photogrammetry camera positioned at $S_{i}$ and it is tracking a

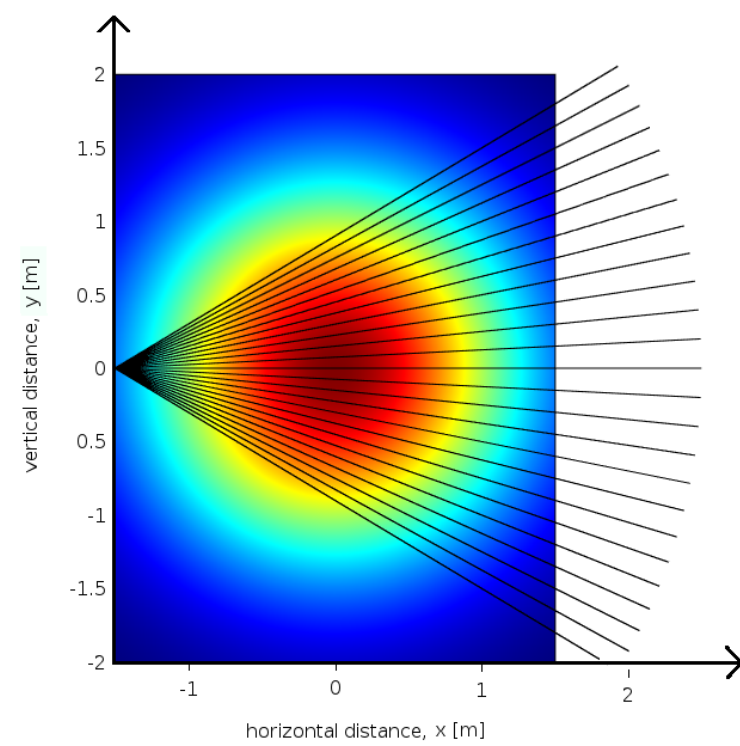

Figure 4. COMSOL simulation of the 25 light rays (black lines) propagating through the Gaussian temperature profile described in (1). The size of the domain is 3 metres by 4 metres in the $x$ and $y$ direction respectively. In this simulation the light rays have starting position $(-1.5,0) \mathrm{m}$ and directional vector $x_{0}=\left[1, y_{0}\right]$ where $y_{0}$ takes the values $0, \pm 0.05, \pm 0.10, \pm 0.15, \ldots, \pm$ 0.6 . reflector at known position $r_{j}$. If we assume that the true temperature distribution of the environment is represented by the Gaussian temperature profile shown, the ray will take the path shown by the solid black line. This temperature distribution causes the light rays to bend towards the heat source (the red colours in Figure 3). As mentioned in Section 2, the photogrammetry system calculates the angle that the line from the camera $\left(c_{i}\right)$ to the reflector $\left(r_{j}\right)$ makes with the normal to the camera plane (denote this angle by $\theta_{i j}$ ). This angle produces a straight line (see the straight dashed line in Figure 3) which shows the equivalent straight ray path that the photogrammetry system uses in its calculations since this assumes the volume is homogeneous. The intersection of the dashed black line and the back wall therefore gives the estimated position of the reflector $r_{i}^{E, c}$ The positional error is then given by $\varepsilon_{2}^{c}=\left|r_{j}-r_{i}^{E, c}\right|$.

The results of the three parametric studies are provided in Figures 5-7. In the first case, the aim was to deduce if the initial ray angle impacted the positional error $\varepsilon^{\mathrm{C}}$. The results (shown in Figure 5) are very intuitive. From this figure we see that as the initial angle of the light ray increase so too does the positional error associated with this. The maximum $\varepsilon_{2}^{c}$ value seen here is approximately $60 \mu \mathrm{m}$ when the ray has an initial angle of approximately $31^{\circ}$. At an initial angle of $0^{\circ}$ the ray does not experience any transverse refractive index gradients. Since it is perpendicular to each isothermal contour, and the transverse temperature gradient is zero then it does not undergo any refraction and so the positional uncertainty is zero.

The second study varied $T_{\max }$ (keeping all other variables constant). This simulation only had one ray propagating through the geometry and the ray that was chosen was the ray whose initial angle led to the largest $\varepsilon_{2}^{c}$ value in the first case.

The results in Figure 6 show that as $T_{\max }$ increases so too does the error $\varepsilon_{2}^{c}$. As $T_{\max }$ increases so too does the local gradient of the temperature (refractive index) profile. It can be seen that the positional uncertainty is now of submillimetre

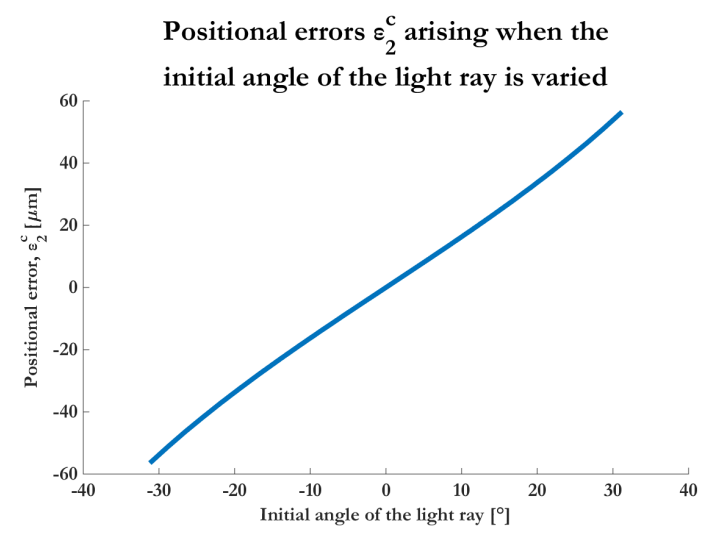

Figure 5. Graph showing the positional errors of the light ray travelling through the Gaussian temperature described in (1) when the initial ray angle is varying. The size of the domain is $3 \mathrm{~m}$ by $4 \mathrm{~m}$ in the $x$ and $y$ direction respectively. There are 25 rays propagating the domain each with a starting position of $(-1.5,0) \mathrm{m}$ and directional vector $x_{0}=\left[1, y_{0}\right]$ where $y_{0}$ takes the values $0, \pm 0.05, \pm 0.10, \pm 0.15, \ldots, \pm 0.6$. The parameters $T_{\max }, T_{\min }$ and $\sigma$ are kept constant throughout at $295 \mathrm{~K}, 314 \mathrm{~K}$ and $1.3107 \mathrm{~m}$ respectively. 


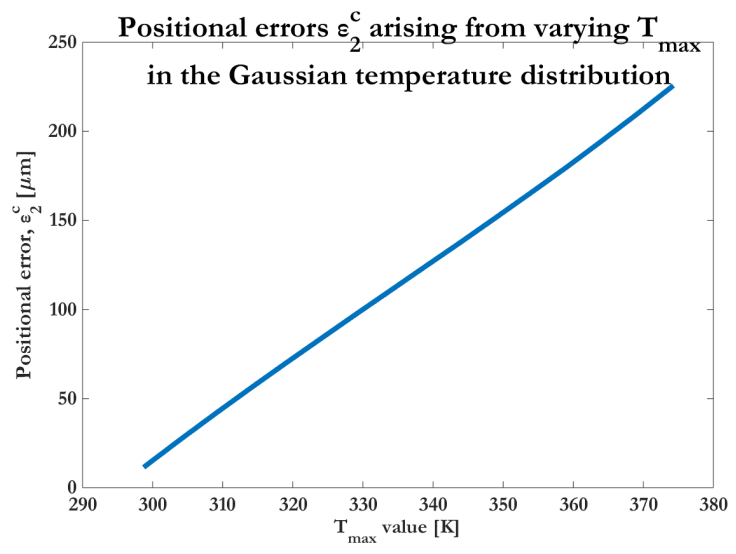

Figure 6. Graph showing the positional errors of the light ray travelling through the Gaussian temperature described in (1) when the range of the temperature profile is varied (via the parameter $T_{\max }$ ). The size of the domain is $3 \mathrm{~m}$ by $4 \mathrm{~m}$ in the $x$ and $y$ direction respectively. The ray in this instance has a directional vector of $x_{0}=[1,0.6]$ as this led to the largest results in the first case. The parameters $T_{\min }$ and $\sigma$ are kept constant throughout at $295 \mathrm{~K}$ and $1.3107 \mathrm{~m}$ respectively. The values for $T_{\max }$ are $299 \mathrm{~K}, 304 \mathrm{~K}, 390 \mathrm{~K}, \ldots, 374 \mathrm{~K}$

\section{Positional Error $\varepsilon_{2}^{c}$ arising from varying the spread $(\sigma)$}

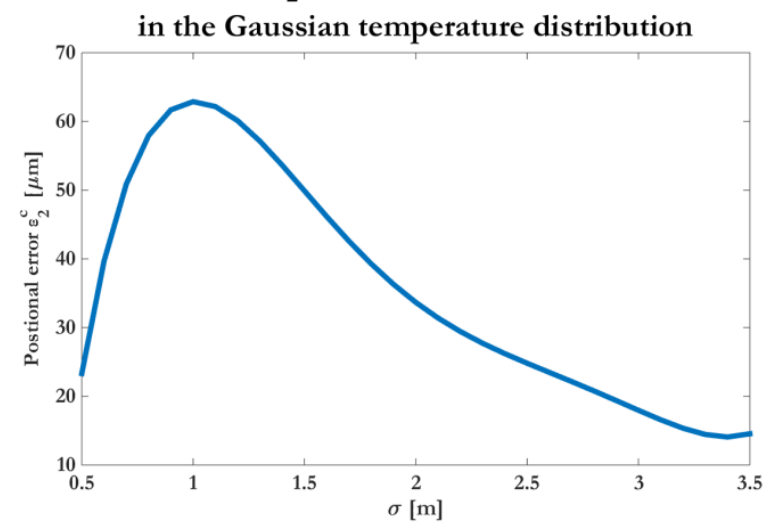

Figure 7. Graph showing the positional errors of the light ray travelling through the Gaussian temperature profile described in (1) when the spread of the profile (as determined via parameter $\sigma$ ) takes the values from 0.5 to 3.5 metres. The size of the domain is $3 \mathrm{~m}$ by $4 \mathrm{~m}$ in the $x$ and $y$ direction respectively. In each of these simulations there is one light-ray propagating through the geometry, with initial position $(-1.5,0) \mathrm{m}$ and directional vector $x_{0}=[1,0.6]$. The variables $T_{\min }$ and $T_{\max }$ are kept constant throughout the simulations taking the values $295 \mathrm{~K}$ and $314 \mathrm{~K}$ respectively.

scale and would start to cause concern in high precision manufacturing setting (over tens of metres this would rise to be of millimetre scale).

Finally, the spread of the temperature profile was varied via the parameter $\sigma$. The results of this are shown in Figure 7. For $\sigma$ values between $0.5 \mathrm{~m}$ and $1.1 \mathrm{~m}$ the positional uncertainty $\varepsilon_{2}^{c}$ value increases, until it reaches a maximum at around $60 \mu \mathrm{m}$ when $\sigma$ is approximately equal to $0.9 \mathrm{~m}$. Thereafter, the $\varepsilon_{2}^{c}$ value decreases as $\sigma$ increases. As $\sigma$ increases the temperature gradient spreads out becoming less steep in the spatial region we are considering, and so it is gradually approaching a constant temperature. So the temperature domain resembles that of a homogeneous one, resulting in reduced refractive index effects and hence a lower $\varepsilon_{2}^{c}$ value. In a similar way when $\sigma$ is decreased the temperature profile narrows until it forms an isolated peak in the spatial domain we are considering. The ray now traverses a domain that is far from this peak and hence there is a very flat temperature profile; so the resulting positional error is significantly reduced.

\section{CONCLUSIONS AND FUTURE WORK}

Light based metrology systems are subject to measurement uncertainty due to refractive index fluctuations in the volume (a heterogeneous refractive index map) which are due primarily to thermal and humidity fluctuations. Over typical measurement distances and temperature profiles found in practice these positional uncertainties can be millimetre scale in the case of photogrammetry. This would suggest that developing methods that can discount the effects of refraction from such measurement systems is essential for their successful deployment in high precision manufacturing environments. Future work will focus on experimentally validating these simulated results. Thereafter, we will deduce if it is possible to use an array of sensors to reconstruct the refractive index map and use this map to discount the refractive effects and therefore reduce the uncertainty in this positioning problem.

\section{ACKNOWLEDGEMENTS}

This work was supported by a studentship from the National Physical Laboratory (NPL), Teddington, London and industry collaboration from Vicon Motion Systems, Oxford, England. The authors would like to thank COMSOL Support, Oxford for their continued support and guidance throughout this work.

\section{REFERENCES}

[1] M. R. Pedersen, L. Nalpantidis, R. S. Andersen, C. Schou, S. Bøgh, V. Krüger and O. Madsen, "Robot skills for manufacturing: From concept to industrial deployment, Robotics and Computer-Integrated Manufacturing", no. 37, (2016), pp.282-291.

[2] H. Park, and N. Tran, "An autonomous manufacturing system for adapting to disturbances", The International Journal of Advanced Manufacturing Technology 56, no. 9 (2011), pp. 11591165.

[3] W. T. Estler, K. L. Edmundson, G. N. Peggs, and D. H. Parker, "Large-scale metrology-an update", CIRP Annals-Manufacturing Technology 51, no. 2 (2002), pp. 587-609.

[4] G. N. Peggs, P. G. Maropoulos, E. B. Hughes, A. B. Forbes, S. Robson, M. Ziebart, and B. Muralikrishnan, "Recent developments in large-scale dimensional metrology", Proc. of the Institution of Mechanical Engineers, Part B: Journal of Engineering Manufacture 223, no. 6 (2009), pp. 571-595.

[5] T. Schenk, "Introduction to photogrammetry." The Ohio State University, Columbus 106 (2005).

[6] A. Lewis et al "LUMINAR: Large volume in industry" [Online] Available at http://projects.npl.co.uk/luminar/the-project/ (2016) [Accessed 28th November 2017].

[7] S. Robson, L. MacDonald, S. Kyle and M.R. Shortis, "Close range calibration of long focal length lenses in a changing environment." ISPRS- International Archives of the Photogrammetry, Remote Sensing \& Spatial Information Sciences, no 41 (2016) pp. 115-122. 
[8] S. Kyle, s. Robson, L. MacDonald and M.R. Shortis, "Compensating for the effects of refraction in photogrammetric metrology" 14 $4^{\text {th }}$ International Workshop on Acceleration Alignment (2016) [online] Available at https://indico.cern.ch/event/489498/contributions/2217509/ [Last Accessed on 11 th December 2017].

[9] R. Summan., S. G. Pierce, C. N. Macleod, G. Dobie, T. Gears, W. Lester, P. Pritchett, and P. Smyth. "Spatial calibration of large volume photogrammetry based metrology systems", Measurement 68 (2015), pp. 189-200.

[10] COMSOL Multiphysics ${ }^{\circledR}$ v. 5.3. www.comsol.com. COMSOL $\mathrm{AB}$, Stockholm, Sweden.

[11] American National Standards Institute B89.4.19-2006, "Performance Evaluation of Laser-based Spherical Coordinate Measurement Systems", American Society of Mechanical Engineers, (2006).
[12] K.M. Nasr, B. Hughes, A. Forbes, and A. Lewis. "Determination of laser tracker angle encoder errors." In EPJ Web of Conferences, vol. 77, (2014) pp. 00002. EDP Sciences.

[13] M. Holá, J. Hrabina, M. Sarbort, J. Oulehla, O. Cíp, and J. Lazar. "Contribution of the Refractive Index Fluctuations to the Length Noise in Displacement Interferometry." Measurement Science Review 15, no. 5 (2015) pp. 263-267.

[14] P.E. Ciddor, "Refractive index of air: new equations for the visible and near infrared." Applied optics 35Ti, no. 9 (1996) pp. 1566-1573.

[15] J.A. Stone and J.H. Zimmerman, "Index of refraction of air: Vacuum wavelength and ambient conditions based on the Ciddor equation", National Institute of Standards and Technology (NIST) [online] Available at: http://emtoolbox.nist.gov/Wavelength/Ciddor.asp [Last Accessed on 28th November 2017]. 